\title{
INTRINSIC CHARACTERIZATIONS OF TANGENT SPACES
}

\author{
HOWARD OSBORN ${ }^{1}$
}

Given a real $C^{\infty}$ structure $A$ on a $C^{\infty}$ manifold containing a point $P$, the tangent space $T_{P}$ is exactly the linear space $E_{P}$ of all linear maps $A \rightarrow^{D} R$ such that $D f g=(P f) D g+(P g) D f$ for $(f, g) \in A \times A$, i.e., the space of $P$-derivations of $A$. However, if $A$ is a $C^{r}$ structure for $r<\infty$ then $E_{P}$ is much larger than $T_{P}$ (see [2] and [3]). Our purpose is to introduce a coordinate-free characterization of $T_{P}$ which is valid for all $r$.

We begin with a short proof of the unpleasant fact that $E_{P}$ is infinite-dimensional for $r=1$. In what follows, a maximal $C^{r}$ structure consists of (globally defined) functions on a $C^{r}$ manifold, including functions whose restrictions to some neighborhood of each point $P$ are coordinate functions, and including every function which is appropriately differentiable in each coordinate patch. Such a restriction is necessary to establish $T_{P}=E_{P}$ in the case $r=\infty$; for example, if $x$ is the usual coordinate function on the real line then the polynomial function algebra in $x$ and $e^{x}$ is clearly not maximal, and in fact there are two linearly independent derivations at each point $P$, given by

$$
\left\{\begin{array} { l } 
{ D _ { 1 } x = 1 } \\
{ D _ { 1 } e ^ { x } = 0 }
\end{array} \text { and } \left\{\begin{array}{l}
D_{2} x=0 \\
D_{2} e^{x}=1 .
\end{array}\right.\right.
$$

Proposition 1. If $A$ is any maximal $C^{1}$ structure then $\operatorname{dim}_{R} E_{P}$ is at least the cardinal of the continuum at each point $P$.

Proof. It suffices to consider the algebra $A$ of all $C^{1}$ functions on the real line, with coordinate function $x \in A$ such that $P x=0$. Let $U$ represent the linear space of all $C^{1}$ functions on the positive real numbers of order $o\left(x^{-1}\right)$ at the origin, $V$ the subspace of $C^{1}$ functions of order $o(1)$ at the origin, and for any $\sigma$ in the open interval $(1,2)$ and $f \in A$ set

$$
\Delta_{\sigma} f=\frac{f-P f}{x^{\sigma}}+V
$$

in the quotient space $U / V$. Since $(f-P f) / x$ and $(g-P g) / x$ are

Presented to the Society, November 14, 1964; received by the editors June 5, 1964.

1 Research supported by National Science Foundation Grant NSF-G-25226. 
bounded at the origin for any $f, g \in A$, the identity

$$
\frac{f g-P f g}{x^{\sigma}}=P f \frac{g-P g}{x^{\sigma}}+P g \frac{f-P f}{x^{\sigma}}+\frac{f-P f}{x} \frac{g-P g}{x} x^{2-\sigma}
$$

implies that $\Delta_{\sigma}$ is a real linear map $A \rightarrow U / V$ such that $\Delta_{\sigma} f g=(P f) \Delta_{\sigma} g$ $+(P g) \Delta_{\sigma} f$, and consequently for any linear functional $\theta$ on $U / V$ such that $\theta(1+V)=1$ the composition $\theta \Delta_{\sigma}$ is a $P$-derivation $D_{\sigma}$ for each $\sigma \in(1,2)$. Since

$$
D_{\sigma}|x| \tau= \begin{cases}0 & \text { if } 1<\sigma<\tau<2 \\ 1 & \text { if } 1<\sigma=\tau<2\end{cases}
$$

and since $|x|^{\tau} \in A$ for $\tau>1$, it follows for each $\tau \in(1,2)$ that $D_{\tau}$ is linearly independent of $\left\{D_{\sigma} \mid \sigma<\tau\right\}$, hence that $\left\{D_{\sigma} \mid \sigma \in(1,2)\right\}$ are mutually linearly independent.

To avoid the difficulty presented by Proposition 1 we let $\gamma A$ denote the continuous functions on the manifold with $C^{1}$ structure $A$, considered as an $A$-module, to obtain the following result:

Proposition 2. For any maximal $C^{1}$ structure $A$, the tensor product over $A$ of $\gamma A$ and the $A$-module of real linear maps $A \rightarrow^{L} \gamma A$ such that $L f g=f L g+g L f$ is exactly the $\gamma A$-module of vector fields.

Proof. For any $f \in A$ the existence of $C^{1}$ Urysohn functions implies that the value $P(L f)$ depends only on the behavior of $f$ in the ring of germs at $P$ (see [1], e.g.), so that we may as well suppose $A$ the algebra of all $C^{1}$ functions on a vector space $W$, with a basis $x^{1}, \cdots$, $x^{n} \in A$ of the linear functionals. Let $W^{-}$and $W^{+}$denote the closed compact subsets

$$
\left\{Q \mid-1 \leqq Q x^{i}-P x^{i} \leqq 0, \quad i=1, \cdots, n\right\}
$$

and

$$
\left\{Q \mid 0 \leqq Q x^{i}-P x^{i} \leqq 1, \quad i=1, \cdots, n\right\},
$$

and for any $f \in A$ let $g_{P}$ denote the restriction of $f$ to $W^{-}$and $h_{P}$ the polynomial function $P f+\sum_{i}\left(P\left(\partial f / \partial x^{i}\right)\right)\left(x^{i}-P x^{i}\right)$ restricted to $W^{+}$. Then by a theorem of Whitney (see [4]) there is a real-valued $C^{1}$ function $f_{P}$ on $W$ whose restrictions to $W^{-}$and $W^{+}$are $g_{P}$ and $h_{P}$, respectively, and which is analytic in the complement. For any derivation $L$ satisfying the hypotheses of the proposition we therefore obtain continuous functions $L f, \sum_{i}\left(P\left(\partial f / \partial x^{i}\right)\right) L x^{i}$, and $L f_{P}$ on $W$ such that $Q L f=Q L f_{P}$ in the interior of $W^{-}$and $Q L f_{P}=\sum_{i}\left(Q L x^{i}\right)\left(P\left(\partial f / \partial x^{i}\right)\right)$ in the interior of $W^{+}$, the value of a derivative at $Q$ depending only on the germs at $Q$. By continuity it follows that $P L f=P L f_{P}$ 
$=\sum_{i}\left(P L x^{i}\right)\left(P\left(\partial f / \partial x^{i}\right)\right)$ for all $P \in W$, and hence that $L f$ $=\sum_{i}\left(L x^{i}\right)\left(\partial f / \partial x^{i}\right)$ for all $f \in A$, which means that $L=\sum_{i}\left(L x^{i}\right)\left(\partial / \partial x^{i}\right)$ as desired.

According to Proposition 2, if $r=1$, the tangent space $T_{P}$ is the subspace of those $P$-derivations of $A$ which can be factored through a derivation of $A$ into the continuous functions, and this is the desired invariant characterization of $T_{P}$. A similar result is valid for any positive integer $r$, the only change in the proof being that one uses polynomials of order $r$ in $x^{i}-y^{i}$ in the definition of $f_{P}$.

We give another description of $T_{P}$ which is simple but unfortunately not invariant since $C^{1}$ norms are defined in terms of "good" derivations given by partial differentiation with respect to some coordinate system.

Let $U$ be any compact coordinate neighborhood of the point $P$ and let \|\|$_{U}$ represent the $C^{1}$ norm of the restriction of a $C^{1}$ structure $A$ to $U$, given by

$$
\|f\|_{U}=\max _{Q \in U}\left\{|Q f|,\left|Q \frac{\partial f}{\partial x^{1}}\right|, \cdots,\left|Q \frac{\partial f}{\partial x^{n}}\right|\right\},
$$

say. A $P$-derivation $D$ is bounded if and only if there is a compact coordinate neighborhood $U$ of $P$ such that $D$ is bounded as a linear operator.

Proposition 3. Let $A$ be a maximal $C^{1}$ structure on a $C^{1}$ manifold containing a point $P$; then $T_{P}$ is the vector space of bounded $P$-derivations.

Proof. For any polynomial function $g$ in $x^{1}, \cdots, x^{n}$ Leibnitz' rule implies $D g=\sum_{i}\left(P\left(\partial g / \partial x^{i}\right)\right) D x^{i}$, and for any $f \in A$ one finds

$$
\begin{aligned}
\left|D f-\sum_{i}\left(P \frac{\partial f}{\partial x^{i}}\right) D x^{i}\right| \leqq & |D f-D g|+\left|D g-\sum_{i}\left(P \frac{\partial g}{\partial x^{i}}\right) D x^{i}\right| \\
& +\sum_{i}\left|P\left(\frac{\partial g}{\partial x^{i}}-\frac{\partial f}{\partial x^{i}}\right) D x^{i}\right| \\
\leqq & \left(\|D\|_{U}+\sum_{i}\left|D x^{i}\right|\right)\|f-g\|_{U}
\end{aligned}
$$

Hence if $\|D\|_{U}<\infty$ one applies the Weierstrass approximation theorem to conclude that

$$
D f=\sum_{i}\left(P \frac{\partial f}{\partial x^{i}}\right) D x^{i}
$$




\section{REFERENCES}

1. A. Frölicher and A. Nijenhuis, Theory of vector-valued differential forms. I, Indag. Math. 18 (1956), 338-359.

2. W. F. Newns and A. G. Walker, Tangent planes to a differentiable manifold, J. London Math. Soc. 31 (1956), 400-407.

3. G. Papy, Sur la définition intrinsèque des vecteurs tangents, C. R. Acad. Sci. Paris 241 (1955), 19-20, ibid. 242 (1956), 1573-1575.

4. H. Whitney, Analytic extensions of differentiable functions defined in closed sets, Trans. Amer. Math. Soc. 36 (1934), 63-89.

UNIVERSITY OF ILLINOIS

\section{THE DUAL OF THE FLABBY IS THE BAR}

JAMES A. SCHAFER

Introduction. The object of this paper is to show the connection between the bar resolution for algebras and the canonical flabby resolution for sheaves. We will show that both resolutions are natural constructions arising from an adjoint relation between two functors. The main result will be that there is a natural chain isomorphism between the "categorical" bar resolution and the dual of the "categorical" flabby resolution.

Throughout this paper $\mathfrak{A}, \mathfrak{B}$ will denote additive categories and $F: \mathfrak{B} \rightarrow \mathfrak{A}, G: \mathfrak{A} \rightarrow \mathfrak{B}$ functors between them with $F$ adjoint to $G$. That is, for each $A$ in $\mathfrak{A}, B$ in $\mathfrak{B}$, there exists a natural isomorphism of abelian groups,

$$
\operatorname{Hom}_{\mathfrak{A}}(F B, A) \cong \operatorname{Hom}_{\mathfrak{B}}(B, G A) .
$$

It follows that there exist natural transformations,

$$
e: I_{\mathfrak{B}} \rightarrow G F, \quad \tilde{e}: F G \rightarrow I_{\mathscr{N}},
$$

with certain properties. An immediate consequence of these properties is that, for all $A$ in $\mathfrak{A}$. we have

$$
G\left(\widetilde{e}_{A}\right) e_{G A}=\operatorname{id}_{G A} .
$$

For this and other results concerning adjoint functors, see [3], [4].

Suppose $S$ and $T$ are functors from the category $\mathfrak{A}$ to the category $\mathscr{B}$ and that $r$ is a natural transformation from $S$ to $T$. If $U$ is a functor from a category $\mathbb{S}$ to a category $\mathfrak{A}$ and $V$ is a functor from a cate-

Received by the editors April 17, 1964. 